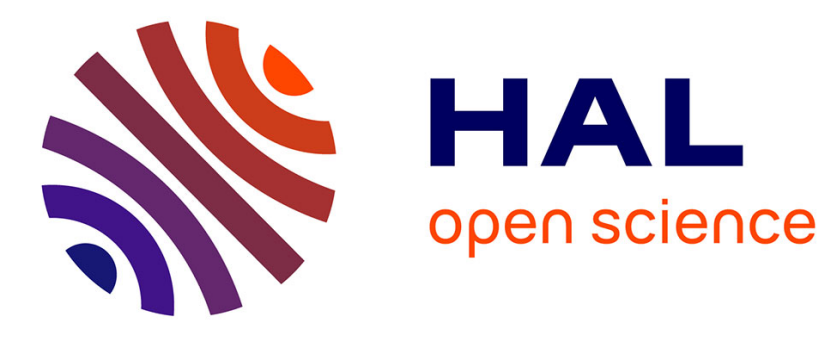

\title{
Random trimer tilings
}

Anandamohan Ghosh, Deepak Dhar, Jesper Lykke Jacobsen

\section{To cite this version:}

Anandamohan Ghosh, Deepak Dhar, Jesper Lykke Jacobsen. Random trimer tilings. Physical Review E: Statistical, Nonlinear, and Soft Matter Physics, 2007, 75, pp.011115. 10.1103/PhysRevE.75.011115 . hal-00093609

\section{HAL Id: hal-00093609 https://hal.science/hal-00093609}

Submitted on 13 Sep 2006

HAL is a multi-disciplinary open access archive for the deposit and dissemination of scientific research documents, whether they are published or not. The documents may come from teaching and research institutions in France or abroad, or from public or private research centers.
L'archive ouverte pluridisciplinaire HAL, est destinée au dépôt et à la diffusion de documents scientifiques de niveau recherche, publiés ou non, émanant des établissements d'enseignement et de recherche français ou étrangers, des laboratoires publics ou privés. 


\title{
Random Trimer Tilings
}

\author{
Anandamohan Ghosh* and Deepak Dhar ${ }^{\dagger}$ \\ Department of Theoretical Physics, \\ Tata Institute of Fundamental Research, \\ Homi Bhabha Road, Mumbai 400 005, India.
}

\author{
Jesper L. Jacobsen ${ }^{\ddagger}$ \\ LPTMS, UMR CNRS 8626, Université Paris Sud, 91405 Orsay, France and \\ Service de Physique Théorique, URA CNRS 2306, CEA Saclay, 91191 Gif sur Yvette, France
}

\begin{abstract}
We study tilings of the square lattice by linear trimers. For a cylinder of circumference $m$, we construct a conserved functional of the base of the tilings, and use this to block-diagonalize the transfer matrix. The number of blocks increases exponentially with $m$. The dimension of the ground-state block is shown to grow as $\left(3 / 2^{1 / 3}\right)^{m}$. We numerically diagonalize this block for $m \leq 27$, obtaining the estimate $S_{\infty}=0.158520 \pm 0.000015$ for the entropy per site in the thermodynamic limit. We present numerical evidence that the continuum limit of the model has conformal invariance. We measure several scaling dimensions, including those corresponding to defects of dimers and $L$-shaped trimers. The trimer tilings of a plane admits a two-dimensional height representation. Monte Carlo simulations of the height variables show that the height-height correlations grows logarithmically at large separation, and the orientation-orientation correlations decay as a power law.
\end{abstract}

PACS numbers:

\section{INTRODUCTION}

The study of statistics of densely packed polymers has long been of interest to physicists. Onsager had argued that solutions of long rod-like molecules should show orientational order at high densities [1]. Flory's approximate analysis suggested that linear rod-like molecules on a two-dimensional lattice should also exhibit an orientational order at high densities [2]. However, for the case of dimers on a lattice - the only case that is analytically soluble - it is known that for all non-zero monomer densities, there is no long-range orientational order [3]. In the limit of zero monomer density, one gets power-law decay of correlations for (bipartite) square and hexagonal lattices [4, 5], but only short-ranged correlations on the triangular lattice [6]. Recently there have been studies of the dimer problem on the cubic lattice [7] and of interacting classical dimers on the square [8] and cubic [9] lattices.

Monte-Carlo simulations of Baumgärtner show that for semi-flexible lattice polymers close to full packing there is no long range order, no phase transition, and the correlation length is of the order of the size of the polymer [10]. However, the exact solution of a single semiflexible polymer that is fully packed on the square lattice (the so-called Flory model) exhibits a low-temperature phase of crystalline order and an infinite-order transition to a disordered, critical high-temperature phase in which the critical exponents vary continuously with temperature [11].

It is generally believed, but not proved, that in the continuum case in three dimensions, long needle-like molecules would undergo a isotropic-nematic transition.

\footnotetext{
*ananda@theory.tifr.res.in

†ddhar@theory.tifr.res.in

$\ddagger$ jesper.jacobsen@u-psud.fr
}

In two dimensions, a spontaneous breaking of continuous rotational symmetry is not allowed, but there is a Kosterlitz-Thouless phase with power-law decay of orientational correlation functions 12, 13]. But the situation is less clear for systems of hard-core molecules on a lattice. In the limit of high density, one can get a solid-like phase where one of the sublattices is preferentially occupied, e.g., in the cases of hard squares and hard hexagons 114. The same behavior is seen in Monte Carlo studies with lattice models of extended hard discs [15]. However, triangular trimers on the triangular lattice can be solved exactly, and do not show a long-range order even at close packing 16]. De Gennes has argued that long straight needles may not show an ordered phase on the square lattice [17. There are not many studies of other molecular shapes in lattice models, as realistic modeling of actual experimental system assemblies of different shaped molecules (e.g., ellipsoids, banana-shaped molecules, etc.) is better done in the continuum space. Tilings by Lshaped trimers and T-shaped tetramers of $m \times \infty$ strips, for $m \leq 5$ have been studied using the transfer matrix technique earlier 18, 19.

In this paper we study random trimer tiling of the square lattice with horizontal and vertical trimers (Fig. 1). We wish in particular to assess if there is a longranged correlation of the orientational order in this problem in the limit of zero monomer density. The problem is first addressed in the geometry of semi-infinite cylinders of size $m \times \infty$. We show how to set up the corresponding transfer matrix and numerically diagonalize it for $m \leq 27$. We use these data to extrapolate to the $m \rightarrow \infty$ limit, determining in particular the entropy per site in a random trimer tiling in the thermodynamic limit. We also prove the existence of a family of matrices which commute with the transfer matrix and show that the transfer matrix decomposes into a number of blocks which is exponentially large in $m$. Our results for the free energy and various correlation functions are consistent with a conformally invariant system of central charge $c=2.15 \pm 0.2$. In 


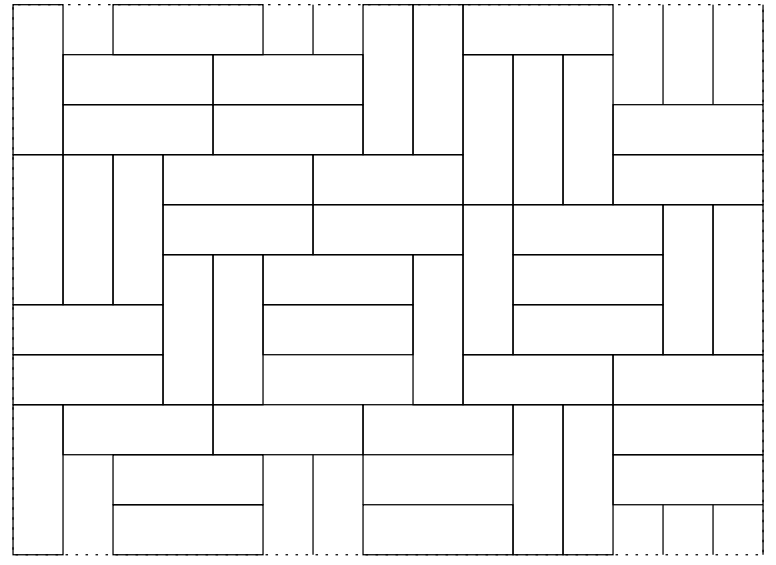

FIG. 1: A trimer tiling with periodic boundary condition in vertical direction.

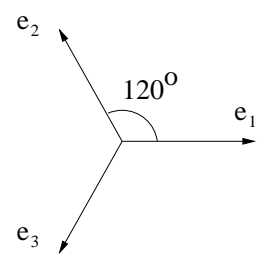

FIG. 2: Choice of unit vectors

particular, the correlation length $\xi_{m}$ is found to increase linearly with $m$.

It is known that trimer tilings admit a two-component height field representation 20]. In the plane, we use this to study correlations of the height field by MonteCarlo simulations, and find that the correlation function $\langle h(\mathbf{r}) h(\mathbf{0}))\rangle$ varies as $\log (|\mathbf{r}|)$ for large $|\mathbf{r}|$. We have also studied the orientational correlations in the simulations, and find that they decay as power laws.

The plan of the paper is as follows: in Section II we present the construction of a height representation for a trimer tiling; in Section III the constants of motion are obtained for trimer tilings on a cylinder; in Section IV the number of disjoint sectors is calculated by the generating function formalism; in Section $\mathrm{V}$ we set up the transfer matrix for the problem, and in Section VI we present the results of numerical diagonalization of the transfer matrix; in Section VII we discuss the trimer-trimer correlation functions and in Section VIII height-height correlation functions, both obtained by Monte-Carlo simulations. Our conclusions are presented in Section IX. An appendix adapts the working of Section IV to a more general tiling problem.

\section{TRIMER TILINGS AND HEIGHT REPRESENTATION}

We represent each trimer as a set of three consecutive squares along a line, oriented horizontally or vertically. For each tiling of a two-dimensional plane by trimers (Fig. 1 ), one can define a configuration of a height model at the vertices of the square lattice, where the heights are twodimensional vectors 21, 22 as follows:
Choose two-dimensional vectors $\mathbf{e}_{1}, \mathbf{e}_{2}$ and $\mathbf{e}_{3}$ such that they satisfy the condition $\mathbf{e}_{1}+\mathbf{e}_{2}+\mathbf{e}_{3}=0$. A convenient choice is $\mathbf{e}_{1}=(1,0), \mathbf{e}_{2}=\frac{1}{2}(-1, \sqrt{3}), \mathbf{e}_{3}=\frac{1}{2}(-1,-\sqrt{3})$, as shown in Fig. 2. Equivalently the vectors can be represented as complex numbers $\mathbf{e}_{1}=1, \mathbf{e}_{2}=\omega$ and $\mathbf{e}_{3}=\omega^{2}$, where $\omega=\mathrm{e}^{2 \pi i / 3}$. The height $\mathbf{h}(i, j)$ at any site $(i, j)$ is an integer linear combination of basis vectors $\mathbf{e}_{1}, \mathbf{e}_{2}$ and $\mathbf{e}_{3}$.

The lattice edges are assumed to be oriented rightwards or upwards, and they are labeled with vectors $\mathbf{e}_{1}, \mathbf{e}_{2}$ and $\mathbf{e}_{3}$ periodically as shown in Fig. 3(a) and (b). The labeling is such that moving along a horizontal or vertical line from any vertex up or right encounters a periodic sequence of labels $\mathbf{e}_{1}, \mathbf{e}_{2}, \mathbf{e}_{3}$. This rule still leaves some freedom in choosing the sequence of bonds; two convenient choices are shown in Figs. 3(a) and (b).

Now, for any given tiling of the square lattice, the height field $\mathbf{h}(i, j)$ is defined so that if the directed edge $\ell$ from site $\mathbf{a}$ to the site $\mathbf{b}$ does not belong to the interior of a tile (i.e., it forms part of the boundary shared by two tiles), and it has label $\mathbf{e}_{\alpha}$, then $h(\mathbf{b})-h(\mathbf{a})=\mathbf{e}_{\alpha}$. This determines the heights at all vertices up to an unimportant additive constant. The constant can be fixed by arbitrarily choosing $h(\mathbf{0})=\mathbf{0}$.

Note that for the two choices of edge-weights shown in Fig. 3, the height difference along any edge of trimer has modulus 1 . For (a), it value is $\sqrt{7}$ or 2 for the internal edges of trimers, and for the choice (b), it only takes the value $\sqrt{7}$. An example of the values of the height field following the convention (a) is illustrated in Fig. 3(c) for a particular configuration of trimers.

The choice (a) has a particular advantage. It can be shown that any trimer covering of a plane can be obtained from any other by a sequence of the basic flip operation, in which three adjacent horizontal trimers are replaced by three adjacent vertical ones (Fig. 14). Under a basic flip move, it is easy to check that the height changes only at four sites and the modulus of the change in height $\|\Delta(\mathbf{h})\|$ is always 3 . We can think of the values of the height field as forming a triangular lattice on the complex plane. This lattice can be broken into 9 sublattices (Fig. 5), such that even after any such flip, the value of height stays on the same sublattice. Also, different sublattices of the height field are in one-to-one correspondence with a 9 sublattice decomposition of the original square lattice (Fig. 5).

\section{CONSTANTS OF MOTION FOR TRIMER TILINGS ON A CYLINDER}

In this section we consider the geometry where the square lattice has been wrapped on a cylinder of circumference $m$. The fully-packing constraint on the trimer tilings then implies strong constraints on the different configurations of trimers along a row of length $m$. In fact, given some local configuration along a row, many local configurations along a different row are disallowed. If we think of the row-to-row transfer matrix as an trimer evolution operator for configurations on a line these constraints can be described in terms of some constants of motion under this evolution. 
(a)

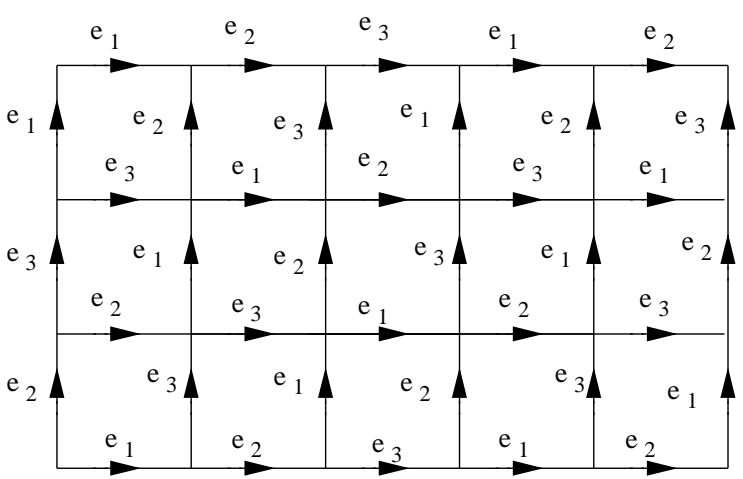

(b)

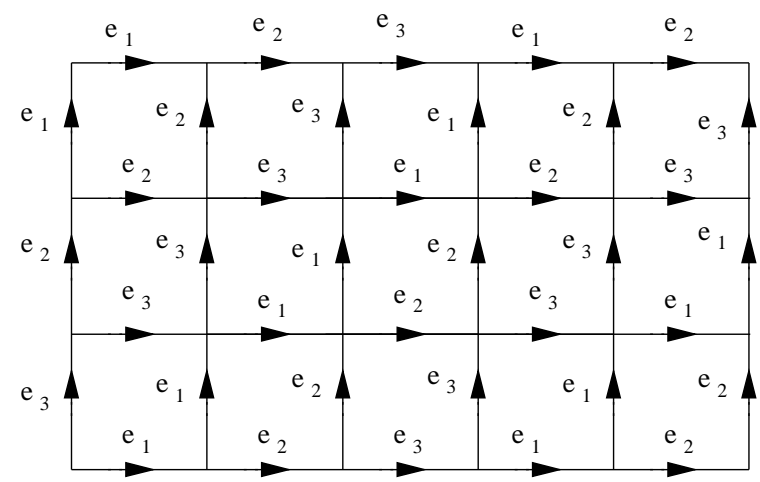

(c)

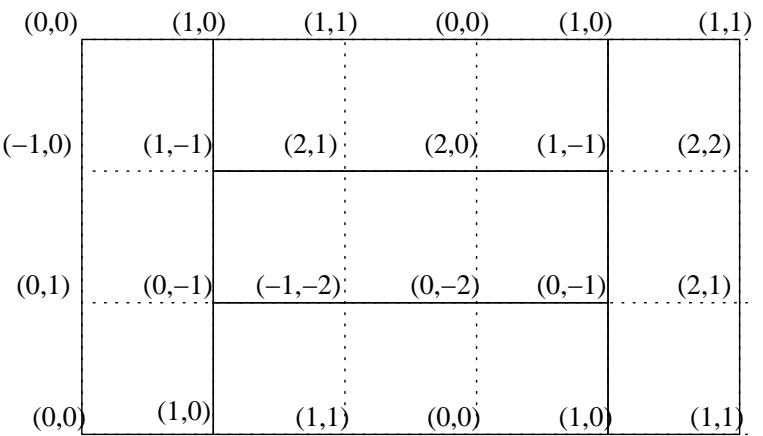

FIG. 3: (a) A trimer tiling of $3 \times 5$ lattice with assignment of labels to the edges of the lattice (b) An alternative assignment of labels (c) the height configuration corresponding to label in (b), where $\left(n_{1}, n_{2}\right)$ denotes the height $\mathbf{h}\left(n_{1}, n_{2}\right) \equiv n_{1} \mathbf{e}_{1}+n_{2} \mathbf{e}_{2}$.

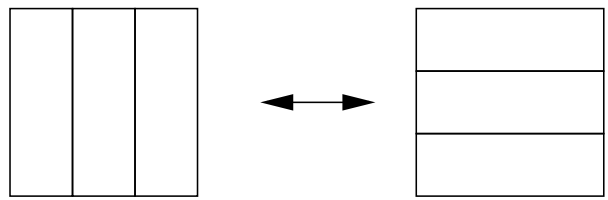

FIG. 4: The flip move exchanging three vertical trimers with three horizontal trimers.

The simplest constant of motion of this type can be constructed in terms of the invariants for loops [23. Given a trimer tiling, define an allowed loop as a sequence of nearest neighbor bonds on the lattice that returns to the starting point, and does not intersect itself, and none of the steps crosses a trimer. In other words, an allowed loop goes along the boundaries of trimers. Define $\mathcal{G}$ as the group generated by two generators $\mathbf{a}$ and $\mathbf{b}$, which (a)

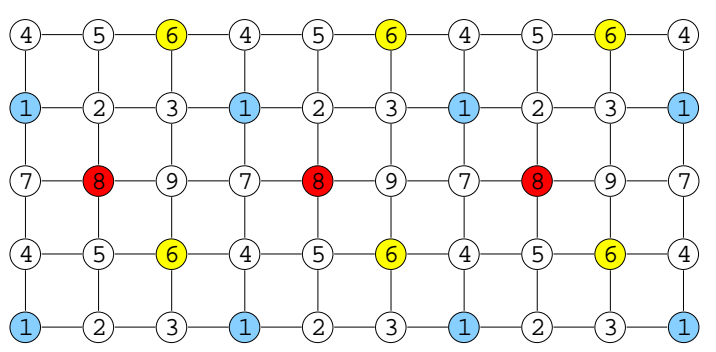

(b)

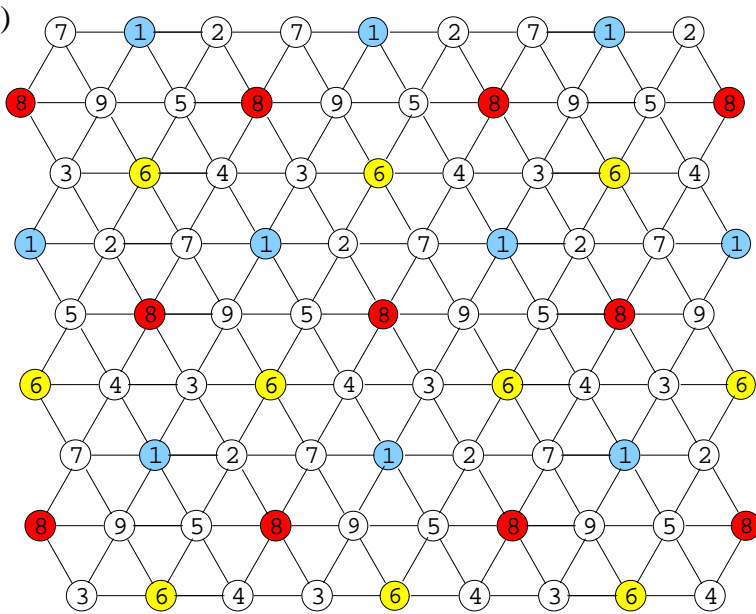

FIG. 5: (a) $3 \times 3$ superlattice decomposition of the square lattice into 9 sublattices. (b) Height variables corresponding to a tiling of the square lattice form a triangular lattice.

satisfy

$$
\begin{gathered}
\mathbf{a}^{3} \mathbf{b}=\mathbf{b a}^{3} \\
\mathbf{a} \mathbf{b}^{3}=\mathbf{b}^{3} \mathbf{a} .
\end{gathered}
$$

Now we attach weights (elements from the group $\mathcal{G}$ ) to each step of the loop. These read respectively $\mathbf{a}, \mathbf{a}^{-1}$, $\mathbf{b}$, and $\mathbf{b}^{-1}$ for a step of the loop to the right, to the left, up, and down. Finally, to each loop $L$ we assign a weight $w_{L} \in \mathcal{G}$ as the ordered product of the weights attached to the steps along the loop. Eqs. (11) imply that $w_{L}$ does not depend on the way (starting point and direction) in which the loop $L$ is traversed when building up the product weight. Moreover, it is easy to see that for a non-winding loop (i.e., an allowed loop on the cylinder which is homotopic to a point), $w_{L}$ is equal to identity. (The proof goes by induction on the number of trimers enclosed by the loop.)

Also, $w_{L}$ takes the same value for any allowed loop that winds around the cylinder. Indeed, let $L$ be such a loop. Then it is easy to see that $w_{L}$ does not change if the loop is deformed locally so that the number of trimers below it changes by \pm 1 . Thus, $w_{L}$ is a constant for the tiling. However, given two products $w_{L}$ and $w_{L^{\prime}}$ of the generators $\mathbf{a}^{ \pm 1}, \mathbf{b}^{ \pm 1}$, checking whether $w_{L}=w_{L^{\prime}}$ by using the rules (1) is nontrivial. We now describe a different construction that is equivalent to this, but more convenient to use.

Fig. 6 shows a partial tiling of the plane, starting from a base (shown hatched in the figure). Each trimer occupies three horizontally or vertically consecutive squares. Periodic boundary conditions in the horizontal $(x)$ direction 
are assumed. The base is supposed without overhangs, and can therefore be specified by its height profile, $H_{0}(x)$. The only constraints about the partial tiling we assume is that it has no holes, and no overhangs. In particular, the tiled region can also be described by a height profile $H(x)$.

We now construct an invariant of the tiling as follows. First, let $\mathbf{A}, \mathbf{B}$ and $\mathbf{C}$ be any three non-commuting matrices satisfying the condition

$$
\mathbf{A}^{3}=\mathbf{B}^{3}=\mathbf{C}^{3} .
$$

Clearly such matrices exist; one family of possible choices is given by

$$
\begin{aligned}
& \mathbf{A}=\left[\begin{array}{lll}
1 & \lambda_{1} & \mu_{1} \\
0 & \omega & \nu_{1} \\
0 & 0 & \omega^{2}
\end{array}\right] \\
& \mathbf{B}=\left[\begin{array}{ccc}
1 & \lambda_{2} & \mu_{2} \\
0 & \omega^{2} & \nu_{2} \\
0 & 0 & \omega
\end{array}\right] \\
& \mathbf{C}=\left[\begin{array}{ccc}
1 & 0 & 0 \\
\lambda_{3} & \omega & 0 \\
\mu_{3} & \nu_{3} & \omega^{2}
\end{array}\right]
\end{aligned}
$$

where $\omega=\mathrm{e}^{2 \pi i / 3}$, and $\lambda_{i}, \mu_{i}, \nu_{i}$ are any complex numbers.

Next, we assign to each square one of the three colors $a$, $b$ or $c$, where each color corresponds to a value of its vertical coordinate $y(\bmod 3)$ (see Fig. 6). The height profile $H(x)$ can then be characterized by a word $l_{1} l_{2} \cdots l_{m}$ over the letters $a, b, c$, where for any $x=1,2, \ldots, m$ the letter $l_{x}$ specifies the color corresponding to $y=H(x)$. For example, the word characterizing the partial tiling in Fig. 6 reads bbbaaabcccbccba. Correspondingly to this word, we construct a functional of $H(x)$, denoted by $\mathcal{J}(H(x))$, which is defined as the trace of a product of matrices $\mathbf{A}, \mathbf{B}, \mathbf{C}$, where each factor is obtained from a letter in the word by replacing $a \mapsto \mathbf{A}, b \mapsto \mathbf{B}, c \mapsto \mathbf{C}$. For example, for the sequence given above, we get the functional

$$
\mathcal{J}(H(x))=\operatorname{Tr}[\mathbf{B B B A A A B C C C B C C B A}] .
$$

Note that by the usual cyclic properties of the trace, the functional depends only on the height profile, and not on the starting point. We can therefore write $\mathcal{J}(H(x))=$ $\mathcal{J}(H)$.

We now have the remarkable theorem:

$$
\mathcal{J}(H)=\mathcal{J}\left(H_{0}\right) .
$$

In other words, $\mathcal{J}$ is the same for all valid partial tilings, grown from the same base, and is equal to its value for the base.

The proof is by induction on the number of trimers in the tiling. It is clearly true for no trimers. If we add a vertical trimer at $x$, the height increases by 3 , and the letter $l_{x}$ does not change. If we add a horizontal trimer, it must be done on a locally flat substrate. Then in the word, the substring aaa may be replaced by $b b b$, or $b b b$ by $c c c$, or $c c c$ by aaa. But by Eq. (2), this does not change J. Q.E.D.

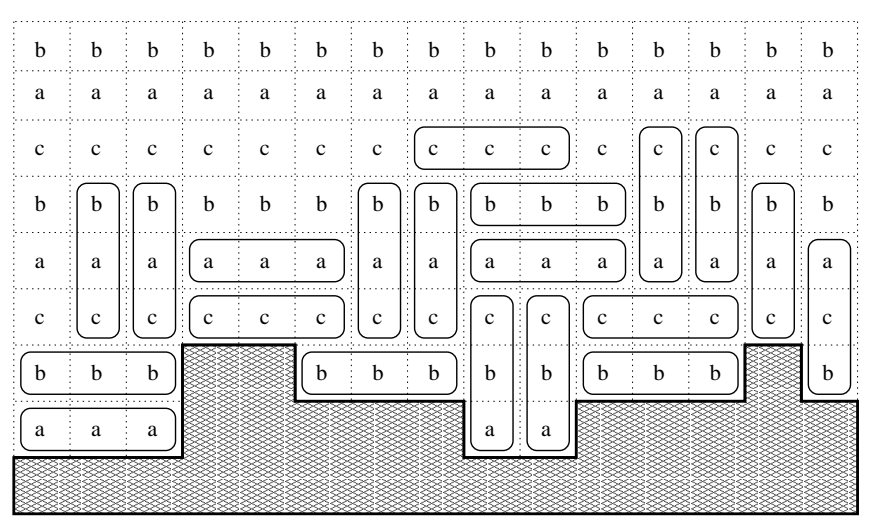

FIG. 6: Partial filling on top of a given base (shown hatched).

For the particular choice of $\mathbf{A}, \mathbf{B}, \mathbf{C}$ given in Eqs. (3), we actually have the additional property $\mathbf{A}^{3}=\mathbf{B}^{3}=$ $\mathbf{C}^{3}=\mathbf{I}$. For any choice of matrices having this additional property the word $l_{1} l_{2} \cdots l_{m}$ can be transformed into an "irreducible word" 24 by recursively deleting any subsequent (modulo $m$ ) occurrences of three equal letters [i.e., $l_{i} l_{i+1} l_{i+2}=a a a$, or $b b b$, or $\left.c c c\right]$ in the original word. In the example shown in Fig. 6 , the base is characterized by the (reducible) word cccbbaaaccaaaba, which then corresponds to the irreducible word bbccba. By the theorem, this is also the irreducible word of any partial tiling built on that base.

The invariant $\mathcal{J}$ is a polynomial function of the parameters $\lambda_{i}, \mu_{i}, \nu_{i}$ in the matrices (3). We can therefore expand $\mathcal{J}$ in multivariable power series in these variables. By the theorem, all the coefficients in this expansion are the same for any allowed tiling, and are constants of motion for the transfer matrix.

For trimers tilings on a torus, we can define other invariants $\mathcal{J}_{\left(n_{x}, n_{y}\right)}$ corresponding to other homotopy classes of non-contractible loops. The allowed homotopy classes are characterized by the winding numbers $n_{x}, n_{y}$ along the two coordinate directions, with $n_{x} \wedge n_{y}=1$ and $n_{x}+n_{y} \geq 1$ 25]. Our previous invariant is $\mathcal{J}=\mathcal{J}_{(1,0)}$. However, the invariants $\mathcal{J}_{\left(n_{x}, n_{y}\right)}$ are not all independent, as can easily be inferred from the simple example when $\mathcal{J}_{(1,0)}$ corresponds to $a b a b a b a b \ldots$; and there is then only one possible trimer tiling which completely fixes the values of all other loop variables.

\section{DECOMPOSITION OF PHASE SPACE INTO DISJOINT SECTORS}

The word associated with the base can be any sequence of $m$ letters chosen from $a, b, c$. The number of all possible base profiles $H_{0}(x)$ (modulo the addition of vertical trimers) for a cylinder of width $m$ is therefore $3^{m}$.

We have already seen that two height profiles $H_{1}(x)$ and $H_{2}(x)$ are reachable from each other if and only if $\mathcal{J}\left(H_{1}(x)\right)=\mathcal{J}\left(H_{2}(x)\right)$. Hence the transfer matrix for this problem has a block diagonal structure, with no transition possible between configurations with different $\mathcal{J}$.

It is straightforward to determine the number of irreducible words of length $n \geq 2$ that start with any two 
specified letters. Let us denote by $N_{d}(n)$ (resp. $\left.N_{s}(n)\right)$ the number of irreducible words in which the initial two letters take fixed different (resp. same) values. Using the condition that in an irreducible word we cannot have any three consecutive letters being identical, it is easy to see that they satisfy the recursion relation

$$
\begin{aligned}
& N_{d}(n+1)=2 N_{d}(n)+N_{s}(n) \\
& N_{s}(n+1)=2 N_{d}(n)
\end{aligned}
$$

for $n \geq 2$, with the initial condition $N_{d}(2)=N_{s}(2)=1$. These equations are easily solved giving

$$
\begin{aligned}
& N_{d}(n)=\frac{1}{4 \sqrt{3}}\left[-(1-\sqrt{3})^{n}+(1+\sqrt{3})^{n}\right] \\
& N_{s}(n)=\frac{1}{12}\left[(3+\sqrt{3})(1-\sqrt{3})^{n}+(3-\sqrt{3})(1+\sqrt{3})^{n}\right]
\end{aligned}
$$

The total number of irreducible strings $t_{n}$ of length $n$ is then obtained by taking into account the multiplicities due to the possible choices of the two initial letters:

$$
t_{n}=6 N_{d}(n)+3 N_{s}(n) .
$$

The total number of sectors for a cylinder of width $m$ (with $3 \mid m$ ) is then $\sum_{k=0}^{m / 3} t_{3 k}$. Note that this number is exponentially large in $m$. For comparison, for dimer tilings (with $2 \mid m$ ) the number of sectors is just $2 m+1$.

Note that each of the $t_{m}$ sectors corresponding to an irreducible word of length $m$ contains only one state of the transfer matrix (these sectors are "stuck"). On the other hand, the $t_{0}=1$ sector corresponding to the empty irreducible word comprises a number of states in the transfer matrix which grows exponentially with $m$.

It is straightforward to obtain precisely this latter number, i.e., the number of words of $n$ letters that reduce to the empty irreducible word. Namely, it corresponds to the dimension of the transfer matrix in the ground state sector, i.e., the sector from which the free energy is obtained.

To this end, let us first define

$$
\begin{aligned}
G_{a} & =a a a+a b b b a a+a a b b b a+a c c c a a \\
& +a a c c c a+a b b b c c c a a+a c c c b b b a a+\ldots
\end{aligned}
$$

as the formal sum over all unfactorizable words with initial letter $a$ that are reducible to the empty word. Here unfactorizable means that the words contributing to $G_{a}$ must not be the concatenation of two non-empty words each of which is in turn reducible to the empty word. Note also that the requirement that the initial letter be $a$ implies, by the property of unfactorizability, that the last reduction before reaching the empty word is of the type $a a a \mapsto \emptyset$. We similarly define $G_{b}$ and $G_{c}$. The sum in Eq. (9) can then be expressed as

$$
\begin{aligned}
G_{a}= & a\left[1+\left(G_{b}+G_{c}\right)+\left(G_{b}+G_{c}\right)^{2}+\cdots\right] \\
& a\left[1+\left(G_{b}+G_{c}\right)+\left(G_{b}+G_{c}\right)^{2}+\cdots\right] a \\
= & a \frac{1}{1-\left(G_{b}+G_{c}\right)} a \frac{1}{1-\left(G_{b}+G_{c}\right)} a .
\end{aligned}
$$

Now substituting $a=b=c=x$ in Eq. (9) we obtain the generating function for irreducible words with a formal weight $x$ per letter:

$$
g(x)=G_{a}(a=x)=\sum_{n=1}^{\infty} g_{3 n} x^{3 n},
$$

where $g_{3 n}$ is the number of different unfactorizable words that start with a given fixed letter and are reducible to the empty word. By Eq. (10), $g(x)$ then satisfies the equation

$$
g(x)[1-2 g(x)]^{2}=x^{3} .
$$

This is a cubic equation in $g(x)$ and can be solved explicitly. Among the three solutions for $g(x)$, two can be discarded as unphysical on the ground that $g(0) \neq 0$. The last, physical solution can be expanded into a polynomial series in $x$, as

$$
\begin{aligned}
g(x) & =x^{3}+4 x^{6}+28 x^{9}+240 x^{12}+2288 x^{15} \\
& +23296 x^{18}+248064 x^{21}+2728704 x^{24}+\cdots .
\end{aligned}
$$

Apart from the trivial root in $x=0, g(x)$ has two nontrivial coincident roots for $x^{3}=x_{\mathrm{c}}^{3}=2 / 27$. For $x$ near $x_{\mathrm{c}}, g(x)$ varies as

$$
g\left(x=x_{\mathrm{c}}-\delta\right)=\frac{1}{6}-A \delta^{1 / 2}+o\left(\delta^{1 / 2}\right) .
$$

This implies that for large $n$

$$
g_{3 n} \sim A\left(\frac{27}{2}\right)^{n} \frac{1}{n^{3 / 2}} .
$$

Using $g(x)$, we can now construct the generating function $H(x)$ of all words (factorizable or not, and with any initial letter) that are reducible to the empty word. We have clearly

$$
\begin{aligned}
H & =1+\left(G_{a}+G_{b}+G_{c}\right)+\left(G_{a}+G_{b}+G_{c}\right)^{2}+\cdots \\
& =\frac{1}{1-\left(G_{a}+G_{b}+G_{c}\right)}
\end{aligned}
$$

Setting $a=b=c=x$ in Eq. (15) as before, we get

$$
H(x)=\sum_{n=1}^{\infty} H_{3 n} x^{3 n},
$$

where $H_{3 n}$ is the total number of words of length $n$ that are reducible to the empty word. The leading terms of the polynomial series are

$$
\begin{aligned}
H(x) & =\frac{1}{1-3 g(x)} \\
& =1+3 x^{3}+21 x^{6}+183 x^{9}+1773 x^{12}+18303 x^{15} \\
& +197157 x^{18}+2189799 x^{21}+24891741 x^{24}+\cdots
\end{aligned}
$$

For $x$ near $x_{\mathrm{c}}, H(x) \approx H\left(x_{\mathrm{c}}\right)-A\left(x_{\mathrm{c}}-x\right)^{1 / 2}$, whence $H_{3 n}$ also varies as

$$
H_{3 n} \sim A\left(\frac{27}{2}\right)^{n} \frac{1}{n^{3 / 2}} .
$$

for large $n$, where $A$ is the same constant as in Eq. (14).

The coefficients appearing in Eq. (17) coincide with the observed dimension of the transfer matrix in the groundstate sector (see Table $\llbracket$ below).

Finally, let us note that the working of this section can be adapted to a more general tiling problem. This is relegated to Appendix A. 


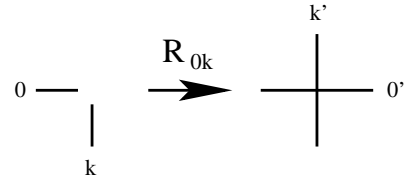

FIG. 7: Labeling of the $R$ matrix.

\section{SETTING UP THE TRANSFER MATRIX}

The number of ways $N_{n}(m)$ to tile a cylinder of width $m$ (with $3 \mid m$ ) and height $n$, with periodic boundary conditions in the $m$-direction and free boundary conditions in the $n$-direction, can be found as $\left\langle 0\left|\left(T_{m}\right)^{n}\right| 0\right\rangle$. Here, $T_{m}$ is the transfer matrix that adds one row of width $m,|0\rangle$ is an initial state corresponding to an initial horizontal base, and $\langle 0|$ is a projection operator on the state corresponding to a final horizontal base. For finite $n$, diagonalizing $T$ by means of a similarity transformation leads to an expression of the form $N_{n}(m)=\sum_{i} \alpha_{i}(m)\left[\lambda_{i}(m)\right]^{n}$, where $\lambda_{i}(m)$ are the eigenvalues of $T$. The corresponding amplitudes $\alpha_{i}(m)$ depend on our choice of boundary conditions in the $n$-direction. We are mainly interested in the limit $n \gg 1$, for which one has simply

$$
N_{n}(m) \approx\left[\lambda_{1}(m)\right]^{n}
$$

where $\lambda_{1}(m)$ is the largest eigenvalue of $T_{m}$. The corresponding entropy per site is then

$$
S_{m}=\frac{1}{m} \log \lambda_{1}(m) .
$$

We shall now describe two different ways of constructing the transfer matrix.

\section{A. First construction}

The first construction is conveniently described in terms of a more general tiling problem (cf. Appendix A) of tiling the plane by horizontal $p$-mers (of size $p \times 1$ elementary squares) and vertical $q$-mers (of size $1 \times q$ ). The trimer case is recovered for $p=q=3$.

We first shift the tiling by half a lattice spacing, both horizontally and vertically, with respect to the underlying square lattice. The tile boundaries then intersect some of the lattice edges, and it is natural to describe the tiling by assigning an appropriate variable ('spin') $s_{i}$ to each lattice edge $i$.

An edge $i$ intersecting a tile boundary has $s_{i}=0$. Each vertical $q$-mer encloses $q-1$ lattice edges which are not intersected by its boundaries; the lowest of these edges has $s_{i}=1$, the second-lowest $s_{i}=2$, and so on, and the highest edge has $s_{i}=q-1$. Each horizontal $p$-mer encloses $p-1$ lattice edges which are not intersected by its boundaries; the leftmost of these edges has $s_{i}=q$, the second-leftmost has $s_{i}=q+1$, and so on, and the rightmost edge has $s_{i}=q+p-2$.

Using a construction well-known from the theory of integrable systems, the row-to-row transfer matrix can be written

$$
T_{m}=\operatorname{Tr}_{0} R_{0 m} \cdots R_{02} R_{01}
$$

where each of the matrices $R_{0 k}$ act on two ingoing edges (labeled 0 and $k$ ), joins them by adding one vertex, and produces two outgoing edges (labeled $k^{\prime}$ and $0^{\prime}$ ), as shown in Fig. 17. The trace over the edge 0 corresponds, graphically, to adding the first horizontal edge of a new row, and making sure that it joins to a horizontal edge carrying the same spin $s_{0}$, once the row has been completed.

It remains to specify the elements of the matrix $R_{0 k}\left(s_{0}, s_{k} ; s_{k}^{\prime}, s_{0}^{\prime}\right)$. These are one for the cases

$$
\left(s_{0}, s_{k} ; s_{k}^{\prime}, s_{0}^{\prime}\right)=\left\{\begin{array}{l}
(0,0 ; 1,0),(0,0 ; q, 0) \\
(0, i ; i+1,0) \text { with } 1 \leq i \leq q-2 \\
(0, q-1 ; 0,0) \\
(i, 0 ; 0, i+1) \text { with } q \leq i \leq q+p-3 \\
(q+p-2,0 ; 0,0)
\end{array}\right.
$$

and zero for all other cases. Indeed, the first line in Eq. (22) corresponds to the lower left corner of any tile; the second line to the interior of a vertical tile; the third line to the upper right corner of a vertical tile; the fourth line to the interior of a horizontal tile; and the fifth line to the upper right corner of a horizontal tile. Finally, the trace in Eq. (21) is over $s_{0}=0, q, q+1, \ldots, q+p-2$.

In some applications it might be of interest to give different weights to horizontal and vertical tiles. This can readily be done, by attributing the desired weight to the lower left corner of each tile, corresponding to the first line of Eq. (22).

The transfer matrix $T_{m}$ is constructed in the base of spins states $\left(s_{1}, s_{2}, \ldots, s_{m}\right)$ corresponding to an initial row of vertical edges, with each $s_{k} \in\{0,1, \ldots, q-1\}$. The factorization (21) of $T_{m}$ is particularly suited for using sparse-matrix and hashing techniques, so that $T_{m}$ can be multiplied onto an arbitrary initial vector in time $\sim$ $m \operatorname{dim}\left(T_{m}\right)$. The first few eigenvalues of $T$ can be found by an iterative scheme (the so-called power method [26]) based on iterating such multiplications.

The ground-state sector, corresponding to growing the tiling from a horizontal base, is obtained by choosing the initial vector so that the reference state $\left(s_{1}, \ldots, s_{m}\right)=$ $(0, \ldots, 0)$ carries weight one, and all other states carry weight zero. The whole state space for the given sector is constructed automatically in the iterative process. For the ground state sector, the total number of states is found to be given by Eq. (17); this constitutes a useful check of the numerical algorithm. Excited sectors, corresponding to non-horizontal bases, can be similarly accessed by choosing another appropriate reference state as the initial vector.

The above construction has enabled us to numerically diagonalize $T_{m}$ for $m \leq 27$. The corresponding eigenvalues are tabulated in Table $\mathbb{\text { I. }}$.

\section{B. Second construction}

The second construction of the transfer matrix is described here for the original trimer tiling problem. We first define the tiling at level $n$ as the set of all tiles that have at least one square with $y$-coordinate less than or equal to $n$. And $H_{n}(x)$ is the height profile for this set of tiles above $y=n$. Then $H_{n}(x)$ clearly lies between 
0 and 2, and may be characterized by a sequence of the type $21222000122110 \cdots$. The transfer matrix $T_{C, C^{\prime}}$ is 1 , if the height configuration $C^{\prime}$ can be reached from $C$ by adding some tiles, otherwise 0 .

The transfer matrix is constructed simply as follows: Let the configuration $C$ be given by some sequence of 0 's, 1 's and 2's as above. We add trimers to this configuration to all sites with height 0 . We can place a horizontal trimer at any place where three consecutive sites have height 0 . This changes the heights at these sites to 1 . We place zero or more horizontal trimers this way. To each remaining site with height 0 we add a vertical trimer, so that the height of that site becomes 3 . Now there are no sites with height 0 , and the maximum height is 3 . Finally we measure heights from a new reference point one unit higher, and decrease all heights by 1 . This gives the new height configuration $C^{\prime}$ with heights given again by a sequence of 0 's, 1's and 2's.

There is a simple way to represent this transfer matrix as a spin hamiltonian. Denote the three possible heights at site $i$ by a quantum spin that can be in any one of three orthonormal states $|0\rangle,|1\rangle$ and $|2\rangle$. We denote the three states at site $i$ by $\left|0_{i}\right\rangle,\left|1_{i}\right\rangle$ and $\left|2_{i}\right\rangle$. Define the operator $S_{i}^{-}$by

$$
S_{i}^{-}\left|h_{i}\right\rangle=\left|h_{i}^{\prime}\right\rangle \text {, where } h-h^{\prime}=1(\bmod 3) .
$$

And we define $P_{i}^{0}$ as the projection operator for the state $\left|0_{i}\right\rangle$, i.e.

$$
P_{i}^{0}\left|0_{i}\right\rangle=\left|0_{i}\right\rangle, \quad P_{i}^{0}\left|1_{i}\right\rangle=P_{i}^{0}\left|2_{i}\right\rangle=0 .
$$

Then it is easy to see that the transfer matrix can be written as

$$
\mathcal{T}=\operatorname{Tr} \prod_{i=1}^{L}\left[\begin{array}{ccc}
S_{i}^{-} & P_{i}^{0} & 0 \\
0 & 0 & P_{i}^{0} \\
P_{i}^{0} & 0 & 0
\end{array}\right]
$$

To prove this, we only need note that expanding the product, the only nonzero terms are of the form $S^{-} S^{-} P^{0} P^{0} P^{0} S^{-} \ldots$, where we have a string of $S^{-}$at consecutive sites, interspersed with the product of three $P^{0}$ 's at consecutive sites.

As an example, let us consider random horizontal and vertical trimer tilings of a square lattice with periodic boundary conditions in the horizontal direction, i.e., an infinite cylinder of width $m$. For convenience we choose $m$ to be a multiple of 3 . A typical tiling of the cylinder is shown in Fig. 1. It is easy to see that on a $3 \times \infty$ cylinder a horizontal trimer can be followed by three vertical trimers, or by another horizontal trimer in exactly three ways due to the periodic conditions in the horizontal direction. The possible height configurations are thus ' 000 ', ' 111 ', and ' 222 ', and the $m=3$ transfer matrix is given by

$$
T=\left[\begin{array}{lll}
3 & 1 & 0 \\
0 & 0 & 1 \\
1 & 0 & 0
\end{array}\right]
$$

We can reduce the size of the transfer matrix by working in a sector where the basis vectors are invariant under translations and reflections. Thus for $6 \times \infty$ cylinder, in the sector where the irreducible word is empty, we have six basis vectors, '000000', '000111', '111111', '000222', '111222', '222222', and the other vectors are related to these by symmetry. The number of vectors needed for the transfer matrix for a $3 n \times \infty$ cylinder is less than $H_{3 n}$ by approximately a factor of $6 n$. The resulting size of the transfer matrix for $n \leq 8$ is shown in Table 1 . By using the rotational and translational symmetries the reduction in the size of the transfer matrix (still in the ground state sector) can be judged by comparing the 2 nd and 3rd columns of Table If. The above construction has enabled us to numerically diagonalize $T_{m}$ for $m \leq 24$. It is less efficient than the sparse matrix obtained by first construction but calculating the nonleading eigenvalues is much easier this way. The corresponding correlation lengths obtained from the second eigenvalue are given in Table I.

We have also studied the case when $m$ is not a multiple of 3 . We recall that in the more familiar case of dimer tilings, for even $m$, there is a one-dimensional height mapping, and accordingly the continuum limit is that of a free boson with $c=1$. For odd $m$, however, the height representation has non-periodic boundary conditions, corresponding to a twist operator, which leads to renormalization of the effective central charge to $c_{\mathrm{eff}}=-2$ 31]. Returning to the trimer problem, we see that taking $m(\bmod 3) \neq 0$ also introduces twist operators here, and would change the effective value of central charge. The entropy per site $S_{m}$ for $m(\bmod 3)=1$ and 2 is listed in Table III.

\begin{tabular}{|r|r|r|r|r|}
\hline$m$ & $\operatorname{dim}_{1}\left(T_{m}\right)$ & $\operatorname{dim}_{2}\left(T_{m}\right)$ & $S_{m}$ & $\xi_{2}$ \\
\hline 3 & 3 & 3 & 0.37754275 & 0.58860147 \\
\hline 6 & 21 & 6 & 0.21764117 & 0.95122814 \\
\hline 9 & 183 & 19 & 0.18163298 & 1.50340426 \\
\hline 12 & 1773 & 99 & 0.17027036 & 2.15126321 \\
\hline 15 & 18303 & 672 & 0.16557863 & 2.83712631 \\
\hline 18 & 197157 & 5667 & 0.16322214 & 3.54463208 \\
\hline 21 & 2189799 & 52689 & 0.16187256 & \\
\hline 24 & 24891741 & 520407 & 0.16102733 & \\
\hline 27 & 288132303 & & 0.16046299 & \\
\hline
\end{tabular}

TABLE I: The dimension of the transfer matrices $T_{m}$, $\operatorname{dim}_{1}\left(T_{m}\right)$, without symmetrization, and $\operatorname{dim}_{2}\left(T_{m}\right)$, with symmetrization, is shown for different $m$. Also shown are the entropy per site $S_{m}$ and the correlation length $\xi_{2}$ defined in Eq. (30).

\section{NUMERICAL DIAGONALIZATION OF THE TRANSFER MATRIX}

Two-dimensional isotropic statistical systems with short-range interactions at the critical point may exhibit invariance under conformal transformations [27]. For a 


\begin{tabular}{|r|r|r|r|}
\hline$m$ & $S_{m}$ & $m$ & $S_{m}$ \\
\hline 4 & 0.15682941 & 5 & 0.13541741 \\
\hline 7 & 0.15773925 & 8 & 0.15031598 \\
\hline 10 & 0.15789911 & 11 & 0.15438343 \\
\hline 13 & 0.15806225 & 14 & 0.15603971 \\
\hline 16 & 0.15817988 & 17 & 0.15687242 \\
\hline 19 & 0.15825951 & 20 & 0.15734824 \\
\hline 22 & 0.15831410 & 23 & 0.15764475 \\
\hline
\end{tabular}

TABLE II: Entropy per site $S_{m}$ for $m(\bmod 3)=1$ and 2 . cylinder of width $m$ the finite-size corrections to the entropy per site $S_{m}$ are then of the form [28]

$$
S_{m}=S_{\infty}+\frac{\pi c}{6 m^{2}}+o\left(m^{-2}\right)
$$

where $S_{\infty}$ is the entropy per site in the thermodynamic limit $m \rightarrow \infty$ (i.e., in the infinite plane) and $c$ is the central charge (conformal anomaly number) which determines the universality class of the problem [27.

\begin{tabular}{|r|r|r|r|r|r|r|r|r|}
\hline$(m, m+3)$ & $S_{\infty}$ & $c$ & $(m, m+3)$ & $S_{\infty}$ & $c$ & $(m, m+3)$ & $S_{\infty}$ & $c$ \\
\hline$(3,6)$ & 0.16434064 & 3.664674 & $(4,7)$ & 0.16728470 & -0.319490 & $(5,8)$ & 0.16121121 & -1.231563 \\
\hline$(6,9)$ & 0.15282642 & 4.456333 & $(7,10)$ & 0.15818038 & -0.041283 & $(8,11)$ & 0.15986633 & -1.167350 \\
\hline$(9,12)$ & 0.15566128 & 4.017785 & $(10,13)$ & 0.15805270 & -0.029334 & $(11,14)$ & 0.15895040 & -1.055394 \\
\hline$(12,15)$ & 0.15723777 & 3.584218 & $(13,16)$ & 0.15829869 & -0.076314 & $(14,17)$ & 0.15871184 & -1.000265 \\
\hline$(15,18)$ & 0.15786649 & 3.314046 & $(16,19)$ & 0.15840838 & -0.111719 & $(17,20)$ & 0.15862738 & -0.968649 \\
\hline$(18,21)$ & 0.15813526 & 3.147735 & $(19,22)$ & 0.15845364 & -0.133847 & $(20,23)$ & 0.15858708 & -0.946406 \\
\hline$(21,24)$ & 0.15826623 & 3.037424 & & & & & & \\
\hline$(24,27)$ & 0.15833844 & 2.957992 & & & & & & \\
\hline
\end{tabular}

TABLE III: Estimates of entropy $S_{\infty}$ and the central charge $c$ from fits using successive pairs of cylinder widths $m$.

It is important that estimates of $S_{\infty}$ obtained by extrapolating of data for different values of $m(\bmod 3)$ have to be consistent with each other. Estimates for $S_{\infty}$ and $c$ obtained by fitting pairs $\left(S_{m}, S_{m+3}\right)$ to Eq. (27) are shown in Table III. Clearly, there is still some residual $m$-dependence. In general, in conformal field theory, one can also have a non-universal $1 / m^{d}$ correction to scaling term in Eq. (27),

$$
S_{m}=S_{\infty}+\frac{\pi c}{6 m^{2}}+\frac{e}{m^{d}},
$$

with $2<d \leq 4$. Here we adopt the following strategy: we choose trial values of $d$ and $S_{\infty}$ and obtain $c$ and $e$ from Eq. 28 by sequential 2-point fits. We find that for different $m$ values there is reasonable convergence for $d=2.78 \pm 0.10$ and $S_{\infty}=0.158520 \pm 0.000015$. In table IV, we have listed the values of $c$ and $e$ obtained by twoterm sequential fits for $S_{m}$ with $m=0,1$ and $2(\bmod 3)$, using the fitting form Eq. (28). The estimate for central charge is $c=2.15 \pm 0.2$. Our estimate for the effective charge is $c_{\text {eff }}=-0.28 \pm 0.02$ for $m=1(\bmod 3)$, and $c_{\text {eff }}=-0.79 \pm 0.02$ for $m=2(\bmod 3)$.

\begin{tabular}{|r|r|r|r|r|r|r|r|r|}
\hline$(m, m+3)$ & $c$ & $e$ & $(m, m+3)$ & $c$ & $e$ & $(m, m+3)$ & $c$ & $e$ \\
\hline$(3,6)$ & 4.48761407 & -0.8859 & $(4,7)$ & -0.11255023 & 0.0932 & $(5,8)$ & -0.77423123 & -0.5984 \\
\hline$(6,9)$ & 2.24822274 & 3.8068 & $(7,10)$ & -0.26174392 & 0.4455 & $(8,11)$ & -0.78830675 & -0.5615 \\
\hline$(9,12)$ & 1.85242965 & 4.9419 & $(10,13)$ & -0.27727375 & 0.4938 & $(11,14)$ & -0.79454721 & -0.5406 \\
\hline$(12,15)$ & 1.98106106 & 4.4810 & $(13,16)$ & -0.27266918 & 0.4762 & $(14,17)$ & -0.79175635 & -0.5519 \\
\hline$(15,18)$ & 2.09438199 & 3.9984 & $(16,19)$ & -0.27314362 & 0.4784 & $(17,20)$ & -0.78906173 & -0.5645 \\
\hline$(18,21)$ & 2.14525639 & 3.7489 & $(19,22)$ & -0.27959514 & 0.5114 & $(20,23)$ & -0.78903295 & -0.5647 \\
\hline$(21,24)$ & 2.15734045 & 3.6821 & & & & & & \\
\hline$(24,27)$ & 2.14936018 & 3.7310 & & & & & & \\
\hline
\end{tabular}

TABLE IV: Estimates of $c$ and $e$ from 2-point fits with $e / m^{d}$ correction for $d=2.774$ and $S_{\infty}=0.15852$ for $m(\bmod 3)=0,1$ and 2 .

To inquire further into the critical behavior of trimer tilings, we can measure as well $S_{m}^{(i)}=\frac{1}{m} \log \lambda_{i}(m)$, where $\lambda_{i}(m)$ is some sub-leading eigenvalue $(i \neq 1)$. From con- 


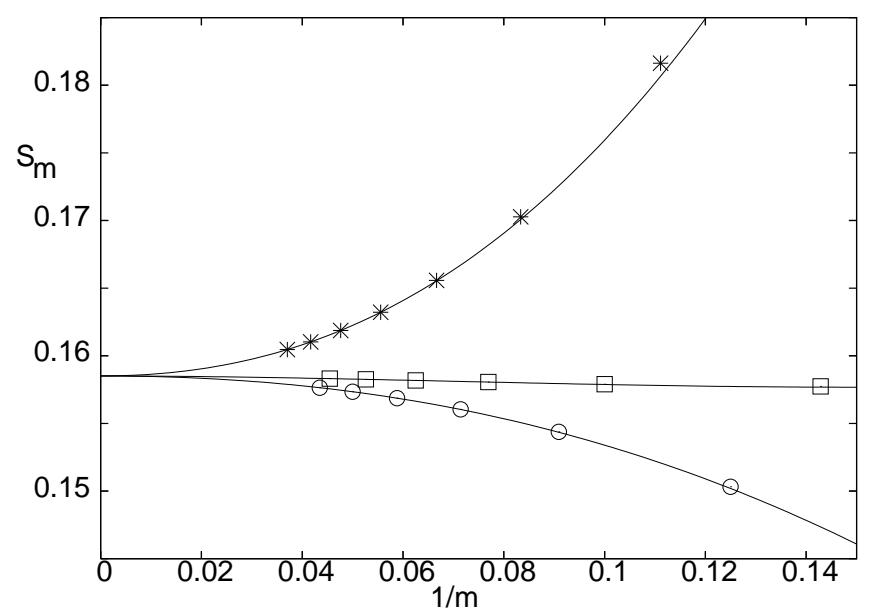

FIG. 8: Convergence of $S_{m}$ with $1 / m$ for $m(\bmod 3)=0(\star)$, $m(\bmod 3)=1(\square), m(\bmod 3)=2(\circ)$. Continuous lines are fits from Eq. 28 that converges to $S_{\infty}=0.15852$ for $d=2.774$ and $c$ and $e$ as listed in Table IV.

formal field theory we expect to get corrections to follow the behavior [29]

$$
S_{\infty}-S_{m}^{(i)}=\left(2 \Delta_{i}-\frac{c}{6}\right) \frac{\pi}{m^{2}}+o\left(m^{-2}\right),
$$

where $\Delta_{i}=h_{i}+\bar{h}_{i}$ is the scaling dimension of the field corresponding to the excited state described by $\lambda_{i}$. Alternatively, Eq. 29) may be stated in terms of the correlation length

$$
\xi_{i}=\frac{1}{\log \left(\lambda_{1} / \lambda_{i}\right)}
$$

of the excitation on the cylinder. This then reads

$$
\xi_{i}=\frac{m}{2 \pi \Delta_{i}},
$$

i.e., the correlation length is proportional to the cylinder width.

We shall now give numerical evidence that Eq. (29) holds true for a number of excitations (i.e., that the resulting finite-size estimates for $\Delta_{i}$ converge as $m \rightarrow \infty$ ). This is support for the hypothesis that trimer tilings are conformally invariant. (We note that Kenyon has proved [30] that dimer tilings are conformally invariant.)

The first kind of excitation $i=2$ in the ground state sector (i.e., with the tilings grown on flat base) has already been mentioned above. The corresponding correlation length $\xi$ is shown in Table 1 and plotted against $m$ in Fig. 9. One finds a slope $a=\frac{1}{2 \pi \Delta_{2}} \simeq 0.23$, corresponding to $\Delta_{2} \sim 0.69$.

We have also studied the case of $3 \mid m$, but with tilings grown from a non-planar base. For simplicity we shall consider only the two simplest sectors. The first one corresponds to taking the initial state $s_{1}=1$, and $s_{k}=0$ for $k=2, \ldots, m$, in the notation of Section $\mathrm{VA}$. The associated exponent $\Delta_{\mathrm{D}}$ describes physically the decay of the correlation function between a pair of widely separated dimer defects in the surrounding soup of trimers. The second sector that we shall consider is built from the

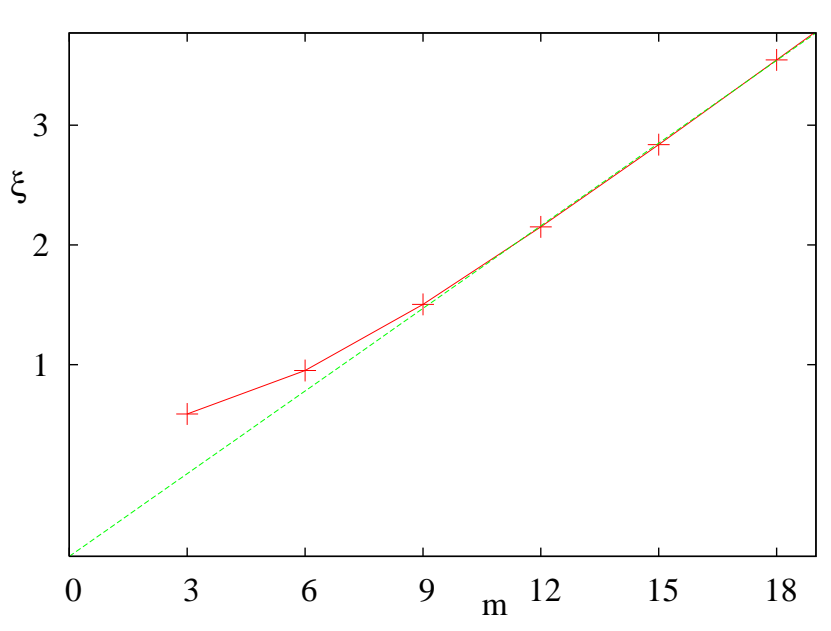

FIG. 9: The correlation length $\xi$ plotted against the cylinder width $m$. The dotted line has the slope $a=0.23$.

\begin{tabular}{|r|r|r|r|r|}
\hline$m$ & $S_{m}^{(D)}$ & $\Delta_{\mathrm{D}}$ & $S_{m}^{(L)}$ & $\Delta_{\mathrm{L}}$ \\
\hline 6 & 0.11284785 & & 0.10663883 & \\
\hline 9 & 0.13675337 & 0.511103 & 0.13215832 & 0.634525 \\
\hline 12 & 0.14560151 & 0.537242 & 0.14225002 & 0.684513 \\
\hline 15 & 0.14988060 & 0.559121 & 0.14733680 & 0.725974 \\
\hline 18 & 0.15229423 & 0.577165 & 0.15029069 & 0.761116 \\
\hline 21 & 0.15379778 & 0.592680 & 0.15217226 & 0.791808 \\
\hline
\end{tabular}

TABLE V: Entropy per site for tiling of non-planar bases and estimates of scaling dimensions with $1 / \mathrm{m}^{d}$ correction with $d=$ 2.774 correction.

initial state $s_{1}=1, s_{2}=2$, and $s_{k}=0$ for $k=3, \ldots, m$. The associated exponent $\Delta_{\mathrm{L}}$ corresponds to a pair of Lshaped defects (or closely bound compounds of a dimer and a monomer).

The estimates for $\Delta_{\mathrm{D}}$ and $\Delta_{\mathrm{L}}$, obtained by adding a non-universal $1 / m^{d}$ correction to Eq. (29), are shown in Table V. The dimer defect is the most relevant. In the continuum limit, described by the two-dimensional height field, the exponents $\Delta_{\mathrm{D}}$ and $\Delta_{\mathrm{L}}$ would describe the decay of vortex-vortex correlation functions. Their final values appear to be of the order $\Delta_{\mathrm{D}}=0.62 \pm 0.1$ and $\Delta_{\mathrm{L}}=0.82 \pm 0.1$, but clearly the words of caution on the slow convergence rate made above when discussing the extrapolations of $c$ are also applicable here.

\section{CORRELATION FUNCTIONS}

Given a trimer tiling, we assign to each lattice face a state $s=1,2, \ldots, 6$ according to its position in the tiling. Our convention is that horizontal trimers are labeled as \begin{tabular}{|lll}
\hline & 2 & 3
\end{tabular} and vertical trimers as $\begin{aligned} & 4 \\
& 5 \\
& 6\end{aligned}$.

Then we can define the correlation function $G_{i j}(\mathbf{x}, \mathbf{Y})$ as the probability that the face at $\mathbf{X}$ is in the state $s=i$ and the face at $\mathbf{Y}$ is in the state $s=j$ (with $1 \leq i, j \leq$ $6)$. In the thermodynamic limit, translational invariance implies that $G_{i j}(\mathbf{X}, \mathbf{Y})$ is only a function of $(\mathbf{X}-\mathbf{Y})$, 
and we write it as $G_{i j}(\mathbf{R})$ with $\mathbf{R}=\mathbf{X}-\mathbf{Y}$. However, all the correlation functions are not independent. For instance, we can express the function $G_{1 j}(\mathbf{R})$ in terms of the function $G_{2 j}(\mathbf{R})$ as

$$
G_{1 j}(\mathbf{R})=G_{2 j}\left(\mathbf{R}+\mathbf{e}_{x}\right) .
$$

Using such equations, one can express the functions where $i$ or $j$ take values $1,3,4$ or 6 in terms of the functions $G_{22}, G_{25}$ and $G_{55}$. Again $G_{22}$ or $G_{55}$ are related by the symmetry in the horizontal and vertical direction. This only leaves the functions $G_{22}$ and $G_{25}$. The conditional probability that the site $\mathbf{R}$ is occupied horizontally, given that the origin is in state 2 , is written $1 / 2+f(\mathbf{R})$, so that the probability of finding a vertical trimer at $\mathbf{R}$ is $1 / 2-f(\mathbf{R})$. We therefore have

$$
\begin{aligned}
& G_{22}(\mathbf{R})+G_{22}\left(\mathbf{R}+\mathbf{e}_{x}\right)+G_{22}\left(\mathbf{R}-\mathbf{e}_{x}\right)=\frac{1}{2}+f(\mathbf{R})(33) \\
& G_{25}(\mathbf{R})+G_{25}\left(\mathbf{R}+\mathbf{e}_{y}\right)+G_{25}\left(\mathbf{R}-\mathbf{e}_{y}\right)=\frac{1}{2}-f(\mathbf{R})(34)
\end{aligned}
$$

The above equations can be solved as linear inhomogeneous equation in $G_{22}$ and $G_{25}$, and can be solved if $f(\mathbf{R})$ is known for all $\mathbf{R}$. Thus we have only one unknown function $f(\mathbf{R})$. Note that Eq. (33) is a difference equation in the $x$-coordinate (the $y$-coordinate does not change). The general solution reads

$$
G_{22}(X, Y)=G_{22}^{0}(X)+A_{Y} \sin k_{0} X+B_{Y} \cos k_{0} X
$$

where $k_{0}=2 \pi / 3$. But $A$ and $B$ must be zero, since the function $G_{22}(X, Y)$ is known to decay to a constant value $1 / 6$ for large $X$. Thus we can determine $G_{22}$ and similarly $G_{25}$ uniquely in terms of $f(\mathbf{R})$.

\section{MONTE-CARLO SIMULATIONS}

We have already introduced the local move of a block of three vertical trimers replacing a block of three horizontal trimers, or vice versa (see Fig. 1). It has been shown that on a square lattice the above move is ergodic, i.e. , all possible configurations can be reached from any initial configuration 22. Starting from any initial configuration, say the standard configuration with all trimers vertical, repeating the local operation at randomly chosen sites generates a random trimer tiling.

In our Monte Carlo simulation, we start with a $L \times$ $L$ lattice fully packed with all trimers vertical $(L=$ $15,45,60,90)$. In one Monte Carlo step, we randomly select one of the trimers, and check if can be flipped using the move of Fig. 4 . If yes, the flip is made, if no, the move is rejected, and another site is selected. We discard the initial $10^{5}$ steps. Once the steady state is reached, we calculate the different correlation functions in the steady state. We generated data for over $10^{5}$ Monte-Carlo steps per site (MCS).

To verify that our sampling produces unbiased results, we have used it to compute the average height on each of the nine different sublattices. These can also be computed analytically as follows. First, we define the absolute values of the heights by setting the mean height $\langle\mathbf{h}\rangle_{1}=0$ on sublattice 1 . To compute $\langle\mathbf{h}\rangle_{2}$ on sublattice 2 , we first note that the height difference between site $\mathrm{A}$, belonging to sublattice 1 , and site $\mathrm{B}$, belonging to sublattice 2, (i.e., along the horizontal edge joining 1 and 2 in Fig. $5($ a $)$ ) can either be 1 if the edge overlaps with the boundary of a horizontal or vertical trimer, or it can be -2 or $2 \omega-\omega^{2}$ if it is one of the internal edges of a vertical trimer. Hence the mean value of $\mathbf{h}_{B}-\mathbf{h}_{A}$ is $(1 / 6)\left(4+2 \omega-\omega^{2}-2\right)=-\omega^{2} / 2$, and since $B$ was arbitrary $\langle\mathbf{h}\rangle_{2}=-\omega^{2} / 2$. In a similar way, we find that

$$
\begin{aligned}
\langle\mathbf{h}\rangle_{1} & =\langle\mathbf{h}\rangle_{5}=\langle\mathbf{h}\rangle_{9}=0 \\
\langle\mathbf{h}\rangle_{2} & =\langle\mathbf{h}\rangle_{6}=\langle\mathbf{h}\rangle_{7}=-\omega^{2} / 2 \\
\langle\mathbf{h}\rangle_{3} & =\langle\mathbf{h}\rangle_{4}=\langle\mathbf{h}\rangle_{8}=\omega / 2 .
\end{aligned}
$$

We have found these values of $\langle\mathbf{h}\rangle_{2}$ and $\langle\mathbf{h}\rangle_{3}$ to be in excellent agreement with the numerical results.

To compute the height-height correlation function

$$
H(x, y)=\left\langle|\mathbf{h}(X+x, Y+y)-\mathbf{h}(X, Y)|^{2}\right\rangle
$$

we averaged the data over all positions $(X, Y)$. The results are shown in Fig. 10. For next nearest neighbors, height difference squared is 1 with probability $2 / 3,4$ and 7 each with probability $1 / 6$. Hence $H(1,0)=H(0,1)=$ $5 / 2$ which has been verified against the results of the simulations. For system size $L, H(x, y)$ varies for large $r$ as

$H(x, y) \approx \Delta_{2} \log \left[(B L)^{2}\left\{\sin ^{2}\left(\frac{\pi x}{L}\right)+\sin ^{2}\left(\frac{\pi y}{L}\right)\right\}+1\right]$

where $\Delta_{2}=0.69$ and $B=4$. Since $H(1,1) \neq H(1,-1)$, there should also be a term depending on $\sin ^{2}(\pi x / L)-$ $\sin ^{2}(\pi y / L)$, but this should decay faster for large $r$, and we have not included it in our fits. For large $r=\sqrt{x^{2}+y^{2}}$ it is easy to see that

$$
H(r) \approx 2 \Delta_{2} \log (r)
$$

or equivalently we have

$$
c-C(r) \approx \Delta_{2} \log (r)
$$

where $c$ is a constant and $C(r)=\langle h(r) h(0)\rangle$. From our estimates of $\Delta_{2}=0.69$ and $a=0.23$, from Eq. (31) it is seen that $\Delta_{2}=1 /(2 \pi a)$ in agreement with the theory of conformal invariance.

In Fig. 11, we have plotted $f(R, \theta)$ for three different directions $\theta=0, \pi / 4, \pi / 2$. We used a lattice of size $90 \times 90$, and $10^{5}$ MCS to get the data. In each case, we see that in each direction, the function $f(R, \theta)$ decreases as a power, $f \sim R^{-x}$, with $x \approx 1.5$. However it is observed that the effective exponent decreases with $R$ and it is difficult to make meaningful estimates due to large fluctuations in the data.

\section{CONCLUSIONS}

We have studied the problem of tiling the plane with trimers, and seen that it differs from the well-known 


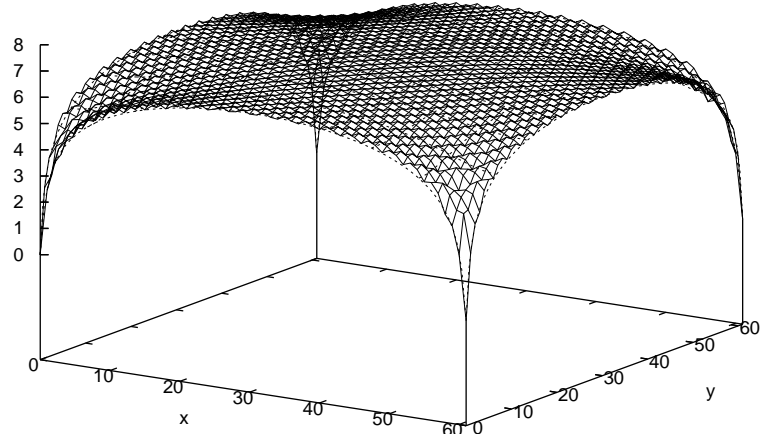

FIG. 10: Correlation function $\mathrm{H}(\mathrm{x}, \mathrm{y})$ for $\mathrm{L}=60$.

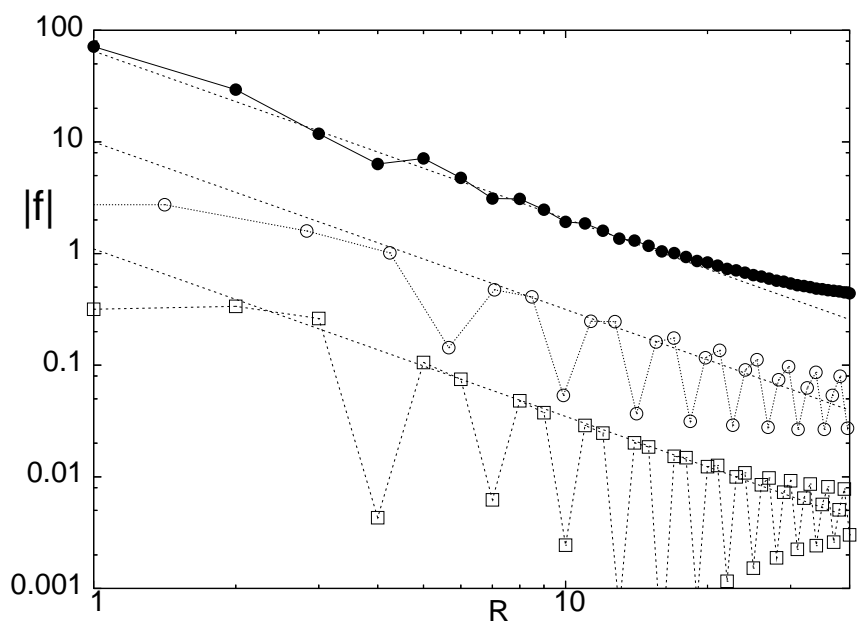

FIG. 11: $|f(R, \theta)|$ as functions of $R$ for $\theta=0(\cdot), \theta=\pi / 4$ (०), $\theta=\pi / 2(\square)$. Straight lines have slope -1.5 .

dimer tiling problem in a number of ways. First, the number of sectors in the transfer matrix grows exponentially rather than linearly with system size. Second, the natural height mapping has a target space dimension which is two rather than one. If the tiling problem is conformally invariant, one could therefore reasonably expect it to have central charge $c=2$. To check this hypothesis, and the inference on $c$, we have performed extensive numerical transfer matrix calculations, on cylinders of widths $m \leq 27$ lattice spacings. The finite-size corrections exhibit unexpectedly large correction-to-scaling terms, making difficult the precise estimations of the critical exponents. The results are however clearly in favor of conformal invariance, and this is confirmed by the logarithmic form of the height-height correlations as obtained by Monte-Carlo simulations. Our estimate $c=2.15 \pm 0.2$ is in marginal agreement with the expectation $c=2$, but we cannot definitely rule out a more complicated behavior. We have also measured numerically a number of other exponents, in particular the leading thermal scaling dimension and the scaling dimensions related to pairs of geometrical defects (dimers and L-shaped trimers).

Acknowledgments. This research has been supported by the Indo-French Centre for the Promotion of Advanced Research (CEFIPRA), project number 3402-2.

\section{APPENDIX A: IRREDUCIBLE WORDS FOR A GENERAL TILING PROBLEM}

The working of section IV can be adapted to a more general tiling problem in which there are two types of straight tiles: horizontal $p$-mers (i.e., of size $p \times 1$ elementary squares) and vertical $q$-mers (of size $1 \times q$ ). We suppose for the rest of this appendix that $p \geq 3$ and that $q \geq 2$.

The words describing the sector decomposition of the transfer matrix are then made of $q$ different letters. An irreducible word is one in which there is no substring of $p$ consecutive equal letters. For $1 \leq k \leq p-1$ we then define $N_{k}(n)$ as the number of irreducible words of length $n$ in which the last $k$ letters are all equal, but in which the last $k+1$ letters are not all equal.

The initial conditions for the recursion relations are by convention

$$
N_{k}(p-1)=1 \text { for } 1 \leq k \leq p-1 \text {. }
$$

This definition of $N_{k}(n)$ does not yet take into account the multiplicity due to the $q$-dependent number of ways that one may chose the last $p-1$ letters. Denoting this multiplicity $M_{k}(p-1)$ one finds

$$
\begin{aligned}
M_{k}(p-1) & =q^{p-1-k}(q-1) \text { for } 1 \leq k \leq p-2 \\
M_{p-1}(p-1) & =q .
\end{aligned}
$$

Indeed, one needs to choose the first $p-1-k$ letters arbitrarily and then complete the word with a single letter, chosen different from the last one chosen arbitrarily. Note that the sum of all multiplicities is $\sum_{k=1}^{p-1} M_{k}(p-1)=$ $q^{p-1}$ as expected.

For any $n \geq p-1$ one then has the recursion relations

$$
\begin{aligned}
N_{1}(n+1) & =(q-1) \sum_{k=1}^{p-2} q^{1-k} N_{k}(n)+q^{3-p} N_{p-1}(n) \\
N_{k}(n+1) & =q N_{k-1}(n) \text { for } 2 \leq k \leq p-2 \\
N_{p-1}(n+1) & =(q-1) N_{p-2}(n) .
\end{aligned}
$$

This generalizes Eq. (6). Equivalently, the recursion relations can be written in matrix form by defining the vector $\vec{N}(n)$ with elements $N_{k}(n)$. One then has $\vec{N}(n+1)=$ $\mathbf{T} \vec{N}(n)$, where $\mathbf{T}=\left\{T_{i j}\right\}$ is a matrix with elements that can be read off from (A3). Iterating this, one finds

$$
\vec{N}(n)=\mathbf{T}^{n+1-p} \overrightarrow{1}=\mathbf{S D}^{n+1-p} \mathbf{S}^{-1} \overrightarrow{1},
$$

where $\overrightarrow{1}=[1,1, \ldots, 1]^{\mathrm{t}}$ and $\mathbf{S}$ is the matrix of eigenvectors of $\mathbf{T}$ that turns $\mathbf{T}$ into diagonal form through $\mathbf{D}=\mathbf{S}^{-1} \mathbf{T S}$. Writing this out for given values of $p$ and $q$ will yield an explicit solution generalizing Eq. (7). 
The total number $t_{n}$ of irreducible words of length $n$ is then obtained by taking into account the multiplicities:

$$
t_{n}=\sum_{k=1}^{p-1} M_{k}(p-1) N_{k}(n)
$$

This generalizes Eq. (8).

To obtain the total number of words that are irreducible to the empty word, we first define $G_{\ell}$ as the number of irreducible unfactorizable words with initial letter $\ell=1, \ldots, q$. Introducing also

$$
G_{0}=\sum_{n=0}^{\infty}\left(\sum_{\ell=2}^{q} G_{\ell}\right)^{n}
$$

the generalization of Eq. (10) reads

$$
G_{\ell}=\ell\left(G_{0} \ell\right)^{p-1} \text {. }
$$

The corresponding generating function $g(x)$ then satisfies

$$
g(x)[1-(q-1) g(x)]^{p-1}=x^{p} .
$$

The generating function for all words that are reducible to the empty word is then

$$
H=\sum_{n=0}^{\infty}\left(\sum_{\ell=1}^{q} G_{\ell}\right)^{n}=\frac{1}{1-\sum_{\ell=1}^{q} G_{\ell}}
$$

and reads explicitly

$$
H(x)=\frac{1}{1-q g(x)}
$$

[1] L. Onsager, Ann. N. Y. Acad. Sci. 51, 627 (1949).

[2] P. J. Flory, Proc. R. Soc. 234, 60 (1956); R. Zwanzig, J. Chem. Phys. 39, 1714 (1963).

[3] O. J. Heilmann and E. Lieb, Comm. Math. Phys. 25, 190 (1972).

[4] P. W. Kasteleyn, Physica 27, 1209 (1961); P. W. Kasteleyn, J. Math. Phys. 4, 287 (1963).

[5] M. E. Fisher and H. Temperley, Phil. Mag. 6, 1061 (1961).

[6] P. Fendley, R. Moessner and S. Sondhi, Phys. Rev. B 66, 214513 (2002).

[7] D. A. Huse, W. Krauth, R. Moessner and S. L. Sondhi, Phys. Rev. Lett. 91, 167004 (2003).

[8] F. Alet, J. L. Jacobsen, G. Misguich, V. Pasquier, F. Mila and M. Troyer, Phys. Rev. Lett. 94, 235702 (2005).

[9] F. Alet, G. Misguich, V. Pasquier, R. Moessner and J.L. Jacobsen, Phys. Rev. Lett. 97, 030403 (2006).

[10] A. Baumgärtner, J. Phys. A: Math. Gen. 17, L971 (1984)

[11] J. L. Jacobsen and J. Kondev, Phys. Rev. Lett. 92, 210601 (2004); Phys. Rev. E 69, 066108 (2004).

[12] D. Frenkel and R. Eppenga, Phys. Rev. A. 31, 1776 (1985).

[13] M. D. Khandkar and M. Barma, Phys. Rev. E 72, 051717 (2005).

[14] R. J. Baxter Exactly solved models in statistical mechanics (Academic Press, London, 1982).

[15] A Bellemans and J Orban, Phys. Rev. Lett. 16, 1038 (1966); J. Chem. Phys. 46, 2922 (1967).

[16] A. Verberkmoes and B. Nienhuis Phys. Rev. Lett. 83, 3986 (1999).

[17] P. G. de Gennes and J. Prost, The physics of liquid crystals (Oxford University Press, 1995) pp. 64-66.

[18] K. Froböse, F. Bonnenmeier and J. Jäckle, J. Phys. A:
Math. Gen. 29, 485 (1996).

[19] C. Moore,

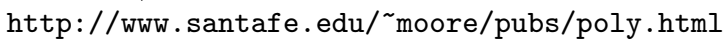

[20] R. Kenyon, Ann. Prob. 28, 759 (2000). Cylindrical boundary conditions for the trimer problem correspond to periodic boundary conditions for the height field only if the width $m$ is divisible by 3 .

[21] C. Moore, http://www . santafe.edu/ moore/pubs/trimer.html

[22] C. Kenyon and R. Kenyon Proc. 33rd Symp. Foundations of Computer Science 610 (1992)

[23] W. P. Thurston, Amer. Math. Monthly 97, 757 (1990).

[24] M. Barma and D. Dhar, Phys. Rev. Lett. 73, 2135 (1994); M. K. Hari Menon and D. Dhar, J. Phys. A: Math. Gen. 28, 6517 (1995).

[25] P. Di Francesco, H. Saleur and J.-B. Zuber, J. Stat. Phys. 49, 57 (1987).

[26] J.H. Wilkinson, The algebraic eigenvalue problem (Oxford University Press, 1988).

[27] J. L. Cardy in Phase Transitions and Critical Phenomena, edited by C. Domb and J. L. Lebowitz (Academic Press, London) 11, 55 (1987).

[28] H.W.J. Blöte, J.L. Cardy and M.P. Nightingale, Phys. Rev. Lett. 56, 742 (1986); I. Affleck, Phys. Rev. Lett. 56, 746 (1986).

[29] J.L. Cardy, Conformal invariance and universality in finite -size scaling, J. Phys. A 17, L385 (1984).

[30] R. Kenyon, Ann. Prob. 29, 1128 (2001)

[31] N. Sh. Izmailian, V. B. Preizzhev, P. Ruelle and C. Hu, Phys. Rev. Lett. 95, 260602 (2005).

[32] H.N.V. Temperley, London Math. Soc. Lecture Notes Series 13, 202 (1974). 